\title{
Anatomical and functional changes in arteries of uterine circulation after modified laparoscopic transabdominal cerclage in pregnancy: A case report and review of the literature
}

\author{
DRAGOS NEMESCU ${ }^{1}$, INGRID ANDRADA TANASA ${ }^{1}$, ROXANA ELENA BOHILTEA ${ }^{2}$, \\ CIPRIAN COROLEUCA ${ }^{2}$, ELVIRA BRATILA ${ }^{2}$ and ANGELA ELENA VINTURACHE ${ }^{3}$ \\ ${ }^{1}$ Department of Obstetrics and Gynecology, 'Grigore T. Popa' University of Medicine and Pharmacy, 700115 Iasi; \\ ${ }^{2}$ Department of Obstetrics and Gynecology, 'Carol Davila' University of Medicine and Pharmacy, \\ 020021 Bucharest, Romania; ${ }^{3}$ Department of Obstetrics and Gynecology, \\ Queen Elizabeth II Hospital, Grande Prairie, AB T8V 2E8, Canada
}

Received April 10, 2020; Accepted May 13, 2020

DOI: $10.3892 /$ etm.2020.8807

\begin{abstract}
Modified laparoscopic transabdominal cerclage (LTAC) was developed as a safer approach for the treatment of cervical insufficiency in pregnancy, with the cerclage tape placed lateral to the uterine vessels. We describe and review the evolution of a woman who successfully underwent an LTAC at 12 weeks of gestation, whose fetus developed growth retardation after 32 weeks. Three-dimensional power Doppler reconstruction viewed both uterine arteries (UtAs) inside of the cerclage until the second trimester. Thereafter, the visualization of UtAs showed the development of a rich array of collateral vascularization, adaptation supported by the findings of low velocity and pulsatility of blood flow in the UtAs. The appearance of the UtAs varies throughout the gestation. There is a moderate variation of the shape and curvature that could result due to their elongation. Furthermore, the cervical length increased during gestation, from 20 to $30 \mathrm{~mm}$. We speculate that this is a mechanical modeling during the late gestation. More studies are needed to understand the hemodynamic and mechanical effects as well as the impact on fetal growth and development of modified LTAC in women with a short cervix or cervical incompetence.
\end{abstract}

\section{Introduction}

Cervical cerclage is used to correct cervical insufficiency in order to prevent second-trimester loss and premature

Correspondence to: Professor Roxana Elena Bohiltea, Department of Obstetrics and Gynecology, 'Carol Davila' University of Medicine and Pharmacy, 169 Splaiul Independenței, 020021 Bucharest, Romania

E-mail: r.bohiltea@yahoo.com

Key words: cervical cerclage, laparoscopy, growth restriction, uterine artery, Doppler, three-dimensional, pulsatility index childbirth. Traditionally, a cerclage is placed via a vaginal approach. Placement of the cervical cerclage via an abdominal approach is recommended in patients with a prior failed vaginal cerclage or with extreme cervical shortening or deformity $(1,2)$. With the advances of minimally invasive surgery, laparoscopic transabdominal cerclage (LTAC) appears to become the preferred treatment option, with a success rate of $79-100 \%$ (3). To overcome the cerclage insertion difficulties in pregnancy, a modified LTAC was developed, where the tape is placed laterally to the uterine vessels (4). However, there is scarce information on the impact of this procedure on the trajectories of the uterine arteries (UtAs) and the uterine-placental circulation. Our experience with a successful modified LTAC performed during the first trimester of pregnancy is reported. The effects of the cerclage on the spatial configuration of the UtAs and of the pulsatility index (PI) along the gestation and fetal growth and development were assessed.

\section{Case report}

Modified laparoscopic transabdominal cerclage. A female patient of 31 years of age, gravida 1, presented for prenatal care to our Institution at 7-week of gestation. The patient provided a signed informed consent agreeing to participate in the study and consented in writing to the anonymized use of information from medical records and the publication of clinical images.

The medical history of the patient was relevant for two cervical conizations for cervical dysplasia. Otherwise, she was healthy. Speculum examination found a small size cervix, flushed to the vagina. The cervical length measured by transvaginal ultrasound was $16 \mathrm{~mm}$. The woman was offered a laparoscopic abdominal cerclage and was counseled on the advantages and risks of the procedure. The cerclage was placed at $11+6$ week gestation, using the modified LTAC technique described by Shin et al (4). Briefly, the woman was placed under general anesthesia, and after the pneumoperitoneum was obtained and the ports were placed, a fenestration was made into the posterior leaf of the broad 


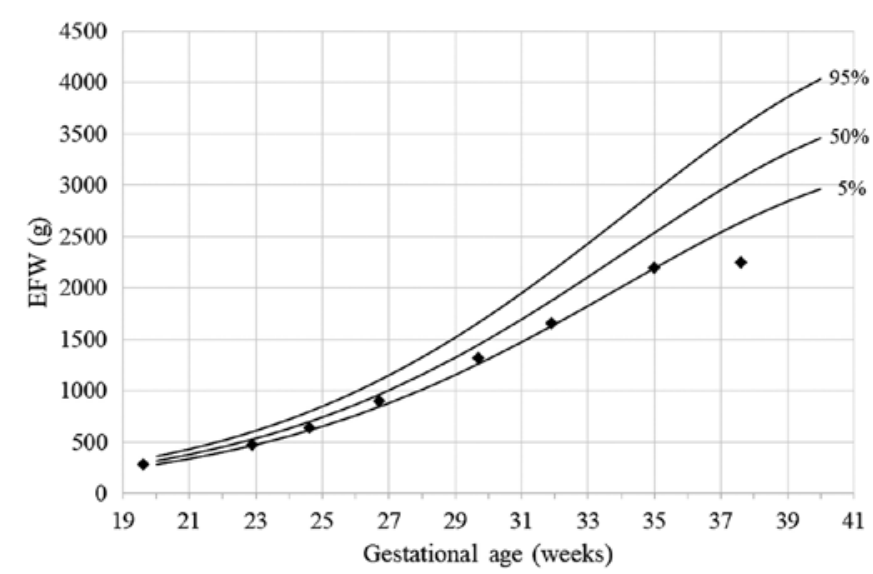

Figure 1. Fetal development throughout pregnancy. The EFW evolved in the second decile until 32 weeks of gestation. A late-onset growth restriction was found at 37 weeks. EFW, estimated fetal weight.

ligament. The peritoneum was incised at the uterovesical fold, and the bladder was reflected downward for complete uterine isthmus exposure. This incision was carried laterally to expose the uterine vessels. A Mersilene tape $(5 \mathrm{~mm}$; Ethicon) was passed through the broad ligament window, laterally to the uterine vessels, with direction from posterior to anterior. The tape was taut until it was flattened along the posterior uterine wall and tied in a knot on the anterior aspect of the uterus. The vesicouterine reflection was reapproximated, covering the Mersilene knot. Placement of the laparoscopic cerclage was uncomplicated, with minimal blood loss. The fetal heart rate was normal before and after the procedure. The patient recovered well after the intervention and was discharged home the following day.

The scan at $12+0$ weeks of gestation found a crown-rump length (CRL) of $56 \mathrm{~mm}$ and a mean UtA pulsatility index (PI) of $2.06(1.199 \mathrm{MoM})$. The mean arterial pressure at 12 weeks was $88 \mathrm{mmHg}(1.083 \mathrm{MoM})$, and PAPP-A was $2.788 \mathrm{IU} / 1$ $(0.842 \mathrm{MoM})$. The woman had an increased calculated risk of preeclampsia and fetal growth restriction before 37 weeks, of 1:103 and 1:49, respectively $(5,6)$. Consequently, she was advised to take $150 \mathrm{mg}$ daily prophylactic dose of aspirin until 35 weeks of gestation. The pregnancy progressed uneventfully, with an estimated fetal weight (EFW) in the second decile, until $32+0$ gestational weeks, when the EFW was $1,660 \mathrm{~g}$, corresponding to the 8th percentile on the fetal growth curve (Fig. 1). From here on, with a diagnostic of small for gestational age (SGA), a serial sonographic assessment was carried for fetal weight, amniotic fluid index, and PI of umbilical artery (UA), middle cerebral artery (MCA), cerebroplacental ratio (CPR), and UtA. The Doppler parameters were normal until 37+4 weeks gestation when the CPR dropped below the 5th percentile (Fig. 2). Oligohydramnios (AFI $2.5 \mathrm{~cm}$ ) and fetal heart rate decelerations were also present. Therefore, emergency cesarean delivery was performed. A female infant with a double nuchal cord, a birth weight of 2,230 g (1st percentile on the growth curve) (7), and an Apgar score of $8 / 9 / 9$ at $1 / 5 / 10$ min was delivered in safe conditions. The infant was admitted to the neonatal unit and experienced no complications. At the time of the cesarean section, the cerclage was left in situ.
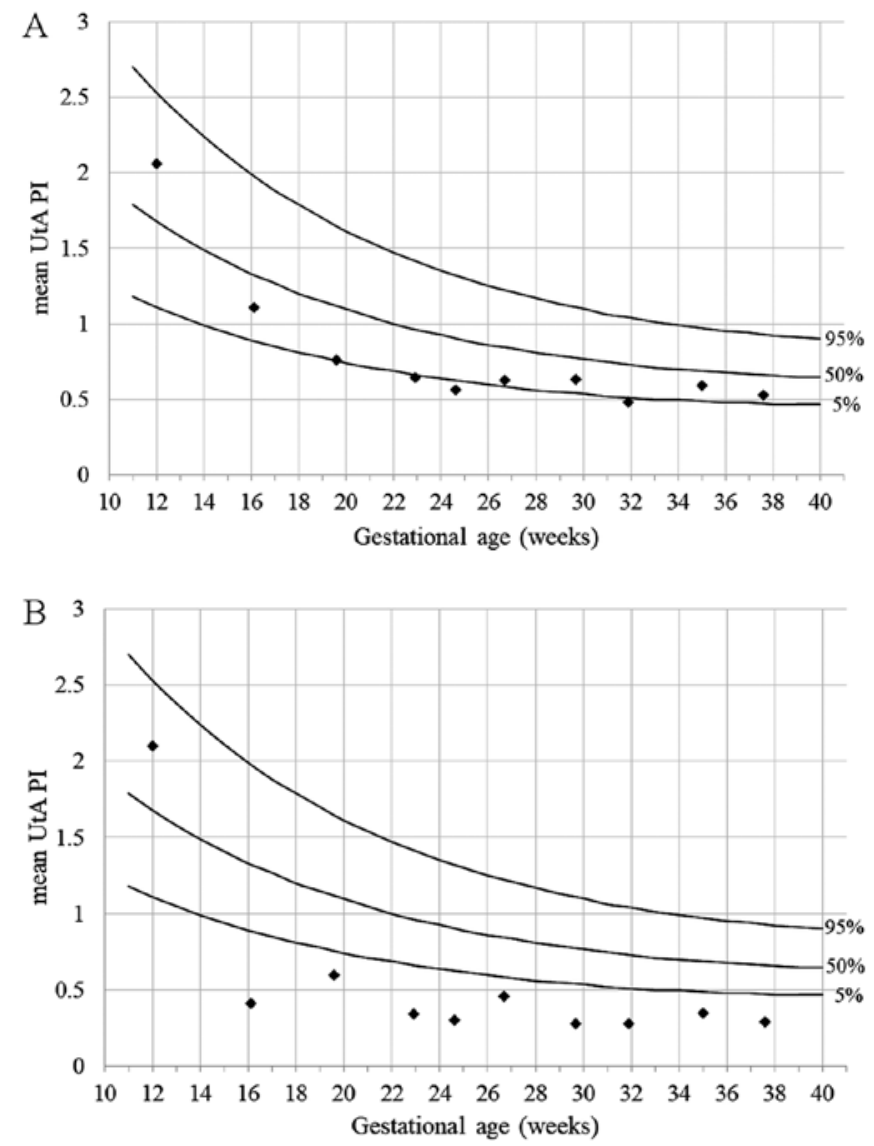

Figure 2. Doppler assessment of the UtA blood flow along the gestation. (A) Mean UtA PI; (B) PI of the left UtA, corresponding to the high vascularization on the placental side. UtA, uterine artery; PI, pulsatility index.

Assessment of maternal-placental-fetal circulation. The appearance of the cerclage was assessed throughout the pregnancy using three-dimensional (3D) ultrasound volumes, as previously described by Ichizuka et al (8). There was no looseness of the tape observed, the internal diameters of the cerclage ring being constant at 26×19 $\mathrm{mm}$. We found, however, that the suture ring rotated soon after the insertion (at 12 weeks scan) to the right such as the knot was located on the right side of the cervix. The distance from the center of the cerclage to the external cervical os increased from 20 to $30 \mathrm{~mm}$ from 12- to 37-weeks' gestation.

The PI of UA and both UtAs were measured at every prenatal consultation until delivery. The UA PI was in the normal ranges throughout the pregnancy. The mean UtA PI was low, varying around the 5th percentile (0.57-0.74 MoM). Notably, the PI of the left UtA, on the side of placental insertion, was markedly low, below the fifth percentile, with a low-velocity flow (Fig. 2).

The UtAs pathway at the level of the abdominal cerclage was evaluated using three-dimensional power Doppler reconstruction. We acquired pelvic power Doppler ultrasound volumes, transvaginally, with a Voluson E10 machine (GE Healthcare) and a RIC 5-9D probe as previously described (9). Following the acquisition, the volume was rotated and translated in a multiplanar display, to obtain an image of the whole tape around the cervix. Then, using the magic cut option, we deleted the gray volume component around, leaving only a thin slice with the cerclage ring in the middle. Additionally, we 

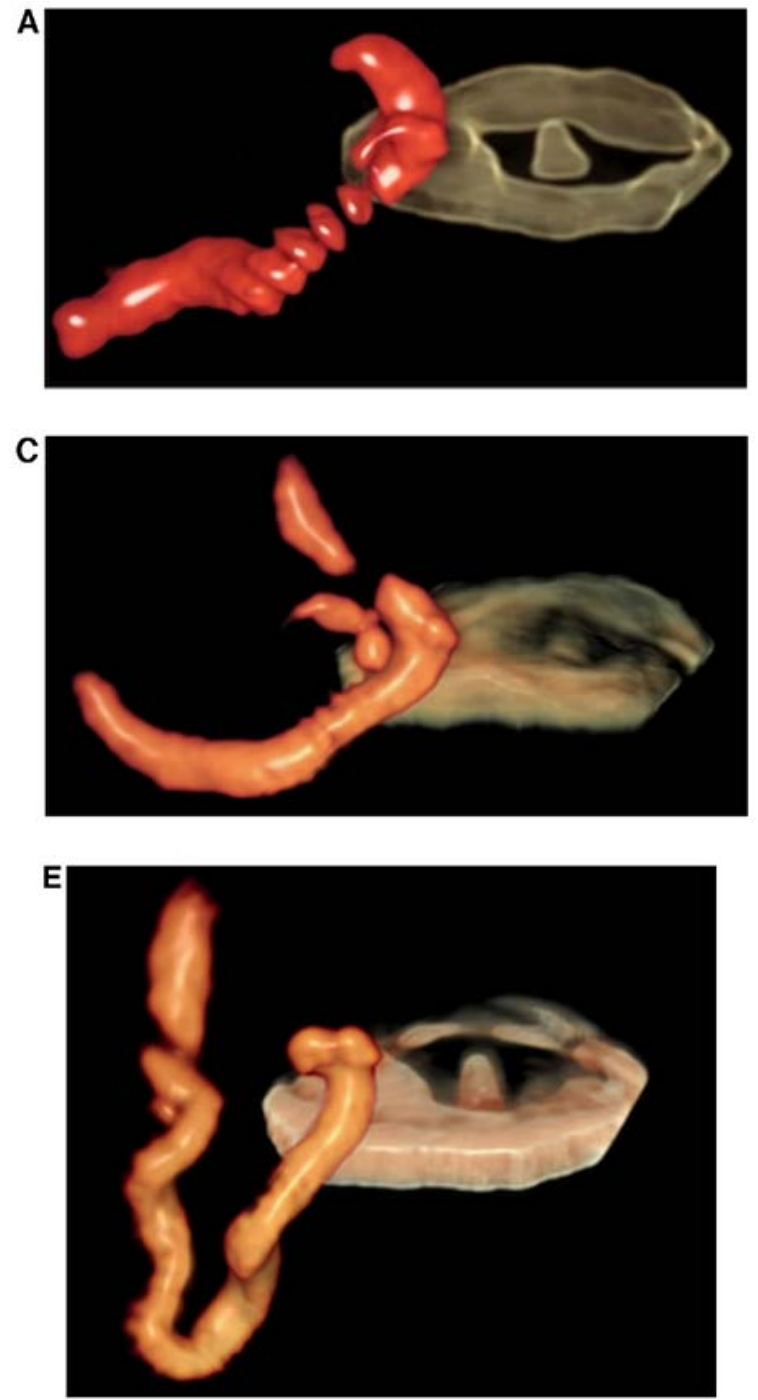
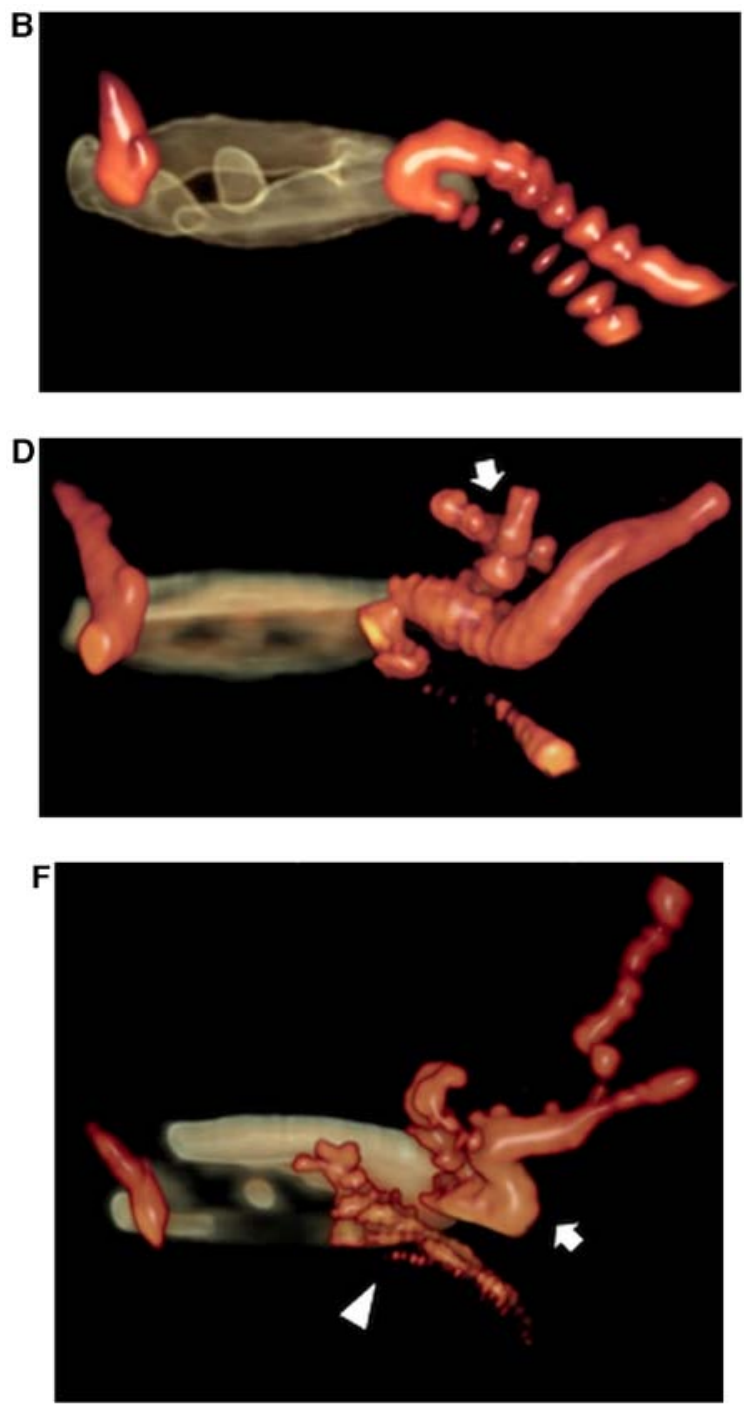

Figure 3. Three-dimensional power Doppler reconstruction of the UtA pathway at the level of the abdominal cerclage. (A) Right UtA at 12 weeks of gestation with an ascending path inside the cerclage ring. (B) Left UtA at 12 weeks of gestation bent over the tape, with an inferior afferent and superior efferent segment. (C) Right UtA at 17 weeks of gestation with an ascending path inside the cerclage ring and a branch (white arrow). (D) Left UtA at 17 weeks of gestation bent over the tape, surrounded by increased vascularization (white arrow) above the ring. (E) Right UtA at 22 weeks, ascending inside the cerclage ring, with a large, deep inferior loop. (F) Left UtA at 22 weeks of gestation with a superior tortuous segment (white arrow), surrounded by other irregular vessels. Below the ring, there is a poor signal from vessels oriented inside the ring (white arrowhead). UtA, uterine artery.

removed all color data not belonging to the UtA from inside of the ultrasound volume. The image was analyzed by glass body rendering in the HD live silhouette mode.

At 12 weeks gestation, 3D power Doppler showed both UtAs passing inside the cerclage ring. The right UtA had a straight ascending pathway, whereas the left UtA was bent, with an ascending and descending segment, apparently pulled by the cerclage (Fig. 3A and B; Videos S1 and S2).

At 17 weeks, the right UtA still had an ascending path, with a new branch to the uterus. The left UtA trajectory maintained the bending at the tape, but there was a reduced view of the ascending branch. The post-tape segment was better visualized, with multiple, enhanced connections of the uterine vasculature shown by the Doppler vascular signals (Fig. 3C and D; Videos S3 and S4).

The anatomy was different at 22 weeks, when the right UtA formed a large, deep loop inferior to the cerclage ring. On the left side, the trajectory of the UtA could not be identified with certainty within the cerclage ring, where there was no Doppler signal. The scan was adjusted to a low threshold for the vascular flow speed (low pulse repetition frequency), which, however, increased the artifacts and the vascular signal around the UtA. There was increased vascularization above the cerclage plane, towards the uterus, but not below the plane, where there was a poor signal from some of the vessels oriented inside of the ring. Analysis of the ultrasound volume was not able to identify any vascular connection between these two zones (Fig. 3E and F; Videos S5 and S6).

After 22 weeks of gestation, the characterization of the configuration of the UtAs was not possible because of the high segmentation of Doppler signal and chaotic vascularization.

\section{Discussion}

A case of a pregnant women who required an abdominal cerclage at 11 weeks gestation due to a short cervix 
diagnosed in the first trimester was reviewd. Our case is unique in that we assessed the impact the cerclage had on the maternal-placental-fetal circulation by measuring the hemodynamic parameters of the flow of UtAs and 3D-visualisation of the remodeling of the uterine circulation.

What is different in our case from the traditionally reported transabdominal cerclages is the modified technique of cerclage insertion. Very few similar cases have been reported in the literature using the modified laparoscopic cervico-isthmic cerclage. However, in those reports, the indication for the modified cerclage was incompetent cervix (10), whereas we chose the method for an early diagnosed short cervix. By placing the suture lateral of the UtAs, one tries to overcome some of the technical challenges of the traditional LTAC performed during pregnancy, aiming to decrease the operational time and morbidity (11). Firstly, there is an increased risk of bleeding due to the highly vascular area close to the UtAs and parametrial veins. The UtAs engorgement makes their skeletonization more difficult compared with the pre-conceptional cerclage (12). Also, the development of UtA windows among gravid patients is more challenging (12). Modified LTAC, in the series reported by Shin et al (4), was associated with lower blood loss, shorter operation time, and postoperative recovery compared with the traditional LTAC. Secondly, the inability to manipulate the gravid uterus poses particular challenges in the traditional cerclage $(4,12)$. Gestational age is an essential factor in the manipulation of the impregnated uterus, and Shin et al showed that no uterine manipulation is required to perform the modified LTAC if done between 11- and 14-weeks of gestation (4). Thirdly, the post-conception cerclage is associated with an increased risk of spontaneous miscarriage and fetal death. First-trimester pregnancy loss was reported as 5\% (4/80) in a modified LTAC series (4), which was similar to the $4.1 \%$ early fetal deaths (before 20 weeks gestation) reported after traditional LAC (13).

However, the effect of the modified LTAC on uterine vessels and fetal development is one of the most significant issues, although sparsely studied to date. Previous reports suggested that this technique does not entirely block the blood flow to the uterus because of an extensive network of collateral connections that provides rich anastomotic communication between different major pelvic vessel systems $(4,10)$. The PI measured at the level of the umbilical artery throughout the gestation, as measured by Seo et al (10) and also reported by us in the present study, support this concept. Furthermore, Shin et al found that the umbilical artery S/D ratio did not vary significantly at 30 and 34 weeks of gestation between the group who underwent modified LTAC and the healthy controls (4).

The effect of cerclage on fetal growth and development is another issue intensely debated in the literature. Using birthweight as a proxy to assess fetal growth and development, Seo et al reported normal fetal growth, with birthweight at the 19th percentile at 37 weeks in a case of modified LTAC (10). In a cohort of 80 women who underwent modified LTAC, Shin et al found that $26 \%$ of cases $(16 / 72)$ had a birth weight below 2,500 g, some of these women also developing severe pregnancy-induced hypertension (4). In our case, the fetus had a relatively low, albeit normal, EFW all along gestation only to develop an SGA status starting at 32 weeks gestation. However, the changes in the maternal-placental-fetal circulation ensued later, at 37 weeks gestation, when abnormal Doppler studies and fetal heart activity, prompted urgent delivery by emergency cesarean section. Our patient, although at risk of preeclampsia, received prophylaxis with low dose aspirin and did not develop gestational hypertension.

In this context, we believe that the course of PI changes at the level of UtAs could be of significance. Starting from 16 weeks of gestation, we found low values of the UtAs mean PI, around the 5th centile. Furthermore, the PI of the UtA on the side of placental insertion (left side in our case) was also very low, less than the 5th centile. This finding is in agreement with previous studies that showed the UtA PI at the placental site to be lower than that at the nonplacental site (14), likely determined by the early development of the uterine collateral circulation, with decreased resistance. Also, low resistance of the uterine circulation could be a sign of uterine congestion, enhanced by the modified placement of the cerclage. Of note, Seo et al found normal values of UtAs mean PI in their reported case (10).

Using three-dimensional power Doppler reconstruction of the UtAs, we show that their appearance varies throughout the gestation. Moderate variation in the shape and curvature could result from the elongation of the UtAs (9) or positional changes of the entire cervical complex within the pelvis. Once a cerclage is placed, this would alter to a certain degree the anatomy of the uterine circulation. Within the vicinity of the cerclage ring, we identified both UtAs from 12 to 17 weeks of gestation. Later in pregnancy, one of the UtAs maintained the typical pathway until delivery, whereas on the other side, where the UtA blood flow was limited, we found a rich vascularization, especially above the plane of the cerclage ring and near the uterus. This vascularization developed from 16 weeks of gestation and could be associated with the progress of the collateral circulation. On the placental side, the low velocity and pulsatility of the blood flow in the left UtA complicated the viewing of the arterial pathway by overlapping the venous flow signal from the surrounding vessels. These characteristics of the blood flow might explain the failure in the visualization of the UtA beyond 22 weeks gestation. Development of varicose veins around the cerclage was recently reported in a 28 weeks pregnancy in a patient with abdominal cerclage inserted after a radical trachelectomy (15).

Finally, we did not find any change in the dimensions or shape of the tape along the gestation, as previously reported (10). We did find, however, that the distance between the external cervical os and the center of the ring increased with $10 \mathrm{~mm}$ by the time of delivery. We speculate that this may represent mechanical modeling of the cervix during the late gestation, the mechanism of which is uncertain. Such cervical changes have not been described before.

In conclusion, we reviewed a successful LTAC at 12 weeks gestation that was followed up with the assessment of growth and maternal-placental-fetal circulation throughout pregnancy. The 3D power Doppler reconstruction viewed both UtAs inside the cerclage ring, and we were able to demonstrate that rich collateral vascularization develops within few weeks from the insertion of the cerclage, as early as 16 weeks of gestation. We found an adaptation of the circulation of UtAs that aims to ensure adequate flow to the placenta. At the same time, there was a significant increase in cervical length during gestation. Whereas, modified LTAC allows a safer technique, the impact of the cerclage on the pregnancy circulation and hemodynamics and fetal growth and development warrants further research. 


\section{Acknowledgements}

Not applicable.

\section{Funding}

No funding was received.

\section{Availability of data and materials}

The data used and/or analyzed during the current study are available from the corresponding author on reasonable request.

\section{Author's contributions}

DN and AEV conceived and designed the study. DN, REB, and IAT provided care to the patient. $\mathrm{CC}$ and $\mathrm{EB}$ performed the surgery. DN and AEV drafted the manuscript. IAT, REB, CC and $\mathrm{EB}$ revised critically the manuscript and made substantial intellectual contributions to the study. All authors read and approved the final version of the manuscript.

\section{Ethics approval and consent to participate}

Patient consent to participate was obtained.

\section{Patient consent for publication}

The patient provided a signed informed consent agreeing to participate in the study. The patient consented in writing to the anonymized use of information from medical records and the publication of clinical images.

\section{Competing interests}

The authors declare that they have no competing interests.

\section{References}

1. Clark NV and Einarsson JI: Laparoscopic abdominal cerclage: A highly effective option for refractory cervical insufficiency. Fertil Steril 113: 717-722, 2020.

2. Shennan A, Chandiramani M, Bennett P, David AL, Girling J, Ridout A, Seed PT, Simpson N, Thornton S, Tydeman G, et al: MAVRIC: A multicenter randomized controlled trial of transabdominal vs transvaginal cervical cerclage. Am J Obstet Gynecol 222: 261.e1-261.e9, 2020.
3. Ades A, Dobromilsky KC, Cheung KT and Umstad MP: Transabdominal cervical cerclage: Laparoscopy versus laparotomy. J Minim Invasive Gynecol 22: 968-973, 2015.

4. Shin SJ, Chung H, Kwon SH, Cha SD, Lee HJ, Kim AR Hwang I and Cho CH: The feasibility of a modified method of laparoscopic transabdominal cervicoisthmic cerclage during pregnancy. J Laparoendosc Adv Surg Tech A 25: 651-656, 2015.

5. Karagiannis G, Akolekar R, Sarquis R, Wright D and Nicolaides KH: Prediction of small-for-gestation neonates from biophysical and biochemical markers at 11-13 weeks. Fetal Diagn Ther 29: 148-154, 2011.

6. O'Gorman N, Wright D, Syngelaki A, Akolekar R, Wright A, Poon LC and Nicolaides KH: Competing risks model in screening for preeclampsia by maternal factors and biomarkers at 11-13 weeks gestation. Am J Obstet Gynecol 214: 103.e101-103.e112, 2016.

7. Nicolaides KH, Wright D, Syngelaki A, Wright A and Akolekar R: Fetal Medicine Foundation fetal and neonatal population weight charts. Ultrasound Obstet Gynecol 52: 44-51, 2018.

8. Ichizuka K, Seo K, Dohi S, Ishikawa T, Sekizawa A and Nagatsuka M: Three-dimensional ultrasound imaging of intra-abdominal cervical-isthmus cerclage. Ultrasound Obstet Gynecol 51: 704-705, 2018.

9. Nemescu D, Navolan DB and Veduta A: Spatial configuration of the uterine artery cervical segment in 3-dimensional reconstruction at 11 to 14 weeks' gestation. J Ultrasound Med 37: 2717-2720, 2018

10. Seo K, Dohi S, Ishikawa T, Ichizuka K, Sekizawa A and Nagatsuka M: Modified laparoscopic cervicoisthmic cerclage in early pregnancy for refractory cervical incompetence: A case report. J Obstet Gynaecol Res 45: 1597-1602, 2019.

11. Whittle WL, Singh SS, Allen L, Glaude L, Thomas J, Windrim R and Leyland N: Laparoscopic cervico-isthmic cerclage: Surgical technique and obstetric outcomes. Am J Obstet Gynecol 201: 364.e361-367, 2009.

12. Moawad GN, Tyan P, Awad C and Abi Khalil ED: Surgical variance between postconceptional and preconceptional minimally invasive transabdominal cerclage placement. Am J Obstet Gynecol 219: 414.e411-414.e412, 2018.

13. Foster TL, Moore ES and Sumners JE: Operative complications and fetal morbidity encountered in 300 prophylactic transabdominal cervical cerclage procedures by one obstetric surgeon. J Obstet Gynaecol 31: 713-717, 2011

14. Kofinas AD, Penry M, Greiss FC Jr, Meis PJ and Nelson LH: The effect of placental location on uterine artery flow velocity waveforms. Am J Obstet Gynecol 159: 1504-1508, 1988.

15. Tamada S, Masuyama H, Hayata K, Eto E, Mitsui T, Eguchi T, Maki J and Tani K: Successful delivery after abdominal radical trachelectomy, using transabdominal cerclage in early pregnancy. Acta Med Okayama 73: 173-176, 2019.

This work is licensed under a Creative Commons Attribution-NonCommercial-NoDerivatives 4.0 International (CC BY-NC-ND 4.0) License. 